\title{
Diffuse trophoblast damage is the hallmark of SARS-CoV-2- associated fetal demise
}

\author{
Marta Garrido-Pontnou $\mathbb{1}^{1} \cdot$ Alexandra Navarro ${ }^{2}$ Jessica Camacho $\mathbb{1}^{2}$ • Fàtima Crispi $\mathbb{1}^{3}$ - Marina Alguacil-Guillén $\left(\mathbb{1}^{4}\right.$. \\ Anna Moreno-Baró ${ }^{2,5}$ - Javier Hernandez-Losa $\mathbb{1}^{2,6} \cdot$ Marta Sesée $^{2,6}$. Santiago Ramón y Cajal $\mathbb{1}^{1} \cdot$ Itziar Garcia Ruíz ${ }^{7}$. \\ Berta Serrano ${ }^{7} \cdot$ Paula Garcia-Aguilar $\mathbb{D}^{7} \cdot$ Anna Suy $^{7} \cdot$ Joan Carles Ferreres $\mathbb{D}^{8} \cdot$ Alfons Nadal $\mathbb{1}^{9,10,11}$
}

Received: 25 March 2021 / Revised: 26 April 2021 / Accepted: 27 April 2021 / Published online: 18 May 2021

(c) The Author(s), under exclusive licence to United States \& Canadian Academy of Pathology 2021

\begin{abstract}
Placental pathology in SARS-CoV-2-infected pregnancies seems rather unspecific. However, the identification of the placental lesions due to SARS-CoV-2 infection would be a significant advance in order to improve the management of these pregnancies and to identify the mechanisms involved in a possible vertical transmission. The pathological findings in placentas delivered from 198 SARS-CoV-2-positive pregnant women were investigated for the presence of lesions associated with placental SARS-CoV-2 infection. SARS-CoV-2 infection was investigated in placental tissues through immunohistochemistry, and positive cases were further confirmed by in situ hybridization. SARS-CoV-2 infection was also investigated by RT-PCR in 33 cases, including all the immunohistochemically positive cases. Nine cases were SARS-CoV-2-positive by immunohistochemistry, in situ hybridization, and RT-PCR. These placentas showed lesions characterized by villous trophoblast necrosis with intervillous space collapse and variable amounts of mixed intervillous inflammatory infiltrate and perivillous fibrinoid deposition. Such lesions ranged from focal to massively widespread in five cases, resulting in intrauterine fetal death. Two of the stillborn fetuses showed some evidence of SARS-CoV-2 positivity. The remaining 189 placentas did not show similar lesions. The strong association between trophoblastic damage and placenta SARS-CoV-2 infection suggests that this lesion is a specific marker of SARS-CoV-2 infection in placenta. Diffuse trophoblastic damage, massively affecting chorionic villous tissue, can result in fetal death associated with COVID-19 disease.
\end{abstract}

These authors contributed equally: Marta Garrido-Pontnou, Alexandra Navarro, Jessica Camacho

These authors jointly supervised this work: Anna Suy, Joan Carles Ferreres, Alfons Nadal

Alfons Nadal

anadal@clinic.cat

1 Pathology Department, Hospital Universitari Vall d'Hebron, Department of Morphological Sciences, School of Medicine, Universitat Autònoma de Barcelona, Barcelona, Spain

2 Pathology Department, Hospital Universitari Vall d'Hebron, Barcelona, Spain

3 Department of Maternal-Fetal Medicine, BCNatal, Barcelona Center for Maternal-Fetal and Neonatal Medicine, Hospital Clínic and Hospital Sant Joan de Déu, IDIBAPS, Universitat de Barcelona, Barcelona, Spain

4 Microbiology Department, Parc Taulí Hospital Universitari. Institut d'Investigació i Innovació Parc Taulí I3PT, Universitat Autònoma de Barcelona, Sabadell, Spain

5 Obstetrics Department, Parc Taulí Hospital Universitari. Institut d'Investigació i Innovació Parc Taulí I3PT, Universitat Autònoma de Barcelona, Sabadell, Spain

\section{Introduction}

COVID-19 dramatically changed the management of many clinical conditions and scenarios. Pregnancy raised particular

6 Pathology Department, Hospital Universitari Vall d'Hebron. Translational Molecular Pathology, Vall d'Hebron Institute of Research (VHIR)., Universitat Autònoma de Barcelona, Barcelona, Spain

7 Obstetrics Department, Hospital Universitari Vall d'Hebron, Barcelona, Spain

8 Pathology Department. Parc Taulí Hospital Universitari. Institut d'Investigació i Innovació Parc Taulí I3PT. Department of Morphological Sciences, School of Medicine, Universitat Autònoma de Barcelona, Sabadell, Spain

9 Pathology Department, Hospital Clínic, Barcelona, Spain

10 Department of Basic Clinical Practice, School of Medicine, Universitat de Barcelona, Barcelona, Spain

11 August Pi i Sunyer Biomedical Research Institute (IDIBAPS), Barcelona, Spain 
concern because SARS-CoV-2 infection complications can affect two different individuals such as the mother and the baby. According to the published literature, placental infection occurs at a low rate estimated in $21 \%$ of pregnancies, but only $2 \%$ of neonates show SARS-CoV-2 positivity [1]. Several reports based largely in placentas delivered by SARS-CoV-2 positive mothers describe different placental pathologic findings in SARS-CoV-2 affected pregnancies. These findings included maternal and fetal vascular malperfusion, perivillous fibrin deposition, and inflammatory lesions such as villitis, intervillitis, and chorioamnionitis. A few fetal deaths were reported associated with inflammatory and ischemic placental findings [2]. However, most of placental findings are not severe and corresponding babies were discharged in good health [3-6].

Recently, a previously unreported placental lesion characterized by trophoblast necrosis was identified in cases with evidence of SARS-CoV-2 placental infection [7-9].

We report here the findings associated with placental SARS-CoV-2 infection that may result in fetal loss depending on the extent of the lesion. We suggest the name of "trophoblastic damage associated with placental SARS-CoV2 infection" for this condition that can display either a focal or a diffuse pattern. This "diffuse trophoblastic damage" is linked with a very poor fetal outcome.

\section{Material and methods}

A total of 198 placentas delivered from SARS-CoV-2-positive pregnant women were submitted for conventional pathological analysis. Sampling was performed according to recommendations of the Amsterdam Consensus for placental sampling and report statements [10]. All placentas were immunohistochemically investigated for the presence of SARS-CoV-2 with the anti-SARS-CoV-2 nucleoprotein (Sino Biological, Wayne, PA) according to manufacturer's instructions. Immunohistochemically positive cases were further analyzed by in situ hybridization (ISH) with the RNAscope-ProbeV-nCoV2019-S (Advanced Cell Diagnostics, Hayward, CA). Thirty-three placentas were investigated for SARS-CoV-2 infection through retrotranscriptase PCR (RT-PCR). Fresh tissue samples and/or swabs were investigated by RT-PCR for SARS-CoV-2 with either the Allplex 2019-nCoV Assay (Seegene, Seoul, South Korea) or the Taqman 2019-nCoV Assay kit v.2 (ThermoFisher Scientific, Waltham, MA, US). For formaldehyde-fixed, paraffin embedded samples, RNA was obtained by High Pure FFPET extraction kit (Roche Diagnostics Gmbh) and further analyzed with CLART ${ }^{\circ}$ COVID-19 (Genomica SAU) following the manufacturer's instructions. Clinical data were retrieved from the institutional records. Significance of association between qualitative variables was tested by Fisher's exact test with an $\alpha$-risk of 0.05 .
Table 1 Association between trophoblastic damage and SARS-CoV-2 infection.

\begin{tabular}{llll}
\hline & \multicolumn{2}{l}{ Trophoblastic damage } \\
\cline { 2 - 3 } & Yes & No & $p$ value \\
\hline $\begin{array}{l}\text { Maternal SARS-CoV-2 positive at } \\
\text { admission for delivery }\end{array}$ & & & $<0.006$ \\
$\quad$ Yes & 7 & 68 & \\
$\quad$ No & 2 & 121 & \\
Placenta SARS-CoV-2 infection & & & $<0.001$ \\
IHC & & & \\
$\quad$ Positive & 9 & 0 & $<0.001$ \\
$\quad$ Negative & 0 & 189 & \\
RT-PCR & & & \\
$\quad$ Positive & 9 & 0 & \\
$\quad$ Negative & 0 & 24 & \\
Total & 9 & 189 & \\
\hline
\end{tabular}

\section{Results}

Immunohistochemistry (IHC) for SARS-CoV-2 showed cytoplasmic positivity in trophoblastic lining in nine cases with histological evidence of trophoblast necrosis. Pattern of staining was patchy both in necrotic and viable trophoblast. SARS-CoV-2 ISH was positive with a similar pattern in the nine immunohistochemically positive cases tested. The nine SARS-CoV-2 positive cases for IHC and ISH were also positive by RT-PCR (Table 1).

The nine immunohistochemically positive placentas showed similar pathologic findings consisting in villous trophoblast necrosis with collapse of the intervillous space. Trophoblast necrosis was continuous, outlining the villi. In contrast, villous stroma was rather spared. Some of the foci showed a moderate amount of intervillous fibrinoid deposition and very variable polymorphous inflammatory infiltrates composed mainly by histiocytes with lower numbers of neutrophils and scattered CD-3 positive T-cell lymphocytes. Small groups of preserved villi were found merged among the areas of trophoblast necrosis. In five cases, this pattern of trophoblast necrosis was diffuse, involving $80-90 \%$ of chorionic tissue. Macroscopically these five placentas had striking gross findings. The placental disks were stiffed and showed increased consistency with a tigroid appearance with white irregular deposits (Fig. 1). These five cases showing a diffuse trophoblastic damage were the only that resulted in fetal loss among the nine cases with SARS-CoV-2-infected placentas $(p=$ 0.0079). Four of the stillborns were submitted for fetal autopsy, showing no significant results. Two of the fetuses had at least one SARS-CoV-2 RT-PCR positive test (lung and nasopharyngeal swab each) (Table 2). The small foci identified in the four placentas from surviving fetuses were 
Fig. 1 Pathology of diffuse trophoblastic damage.

A Grossly the placenta is pale and stiffed. B Histologically, there is diffuse collapse of intervillous space resulting in close contact among chorionic villi with necrosis of trophoblast that stains homogeneously with eosin (H\&E, original magnification 20x). C At higher power nuclear signs of cell necrosis can be readily seen in the involved trophoblast whereas villous stroma is spared (H\&E, original magnification 60x). D Occasional groups of preserved chorionic villi are totally surrounded of villi involved by diffuse trophoblastic damage (H\&E, original magnification $10 \mathrm{x}$ ).

E immunohistochemistry for SARS-CoV-2 shows patchy trophoblast staining (original magnification 30x). F in situ hybridization shows a granular pattern of staining in trophoblast lining of chorionic villi (original magnification 30x).

Table 2 Clinicopathologic features of feto-maternal dyads with SARS-CoV-2 infected placentas.

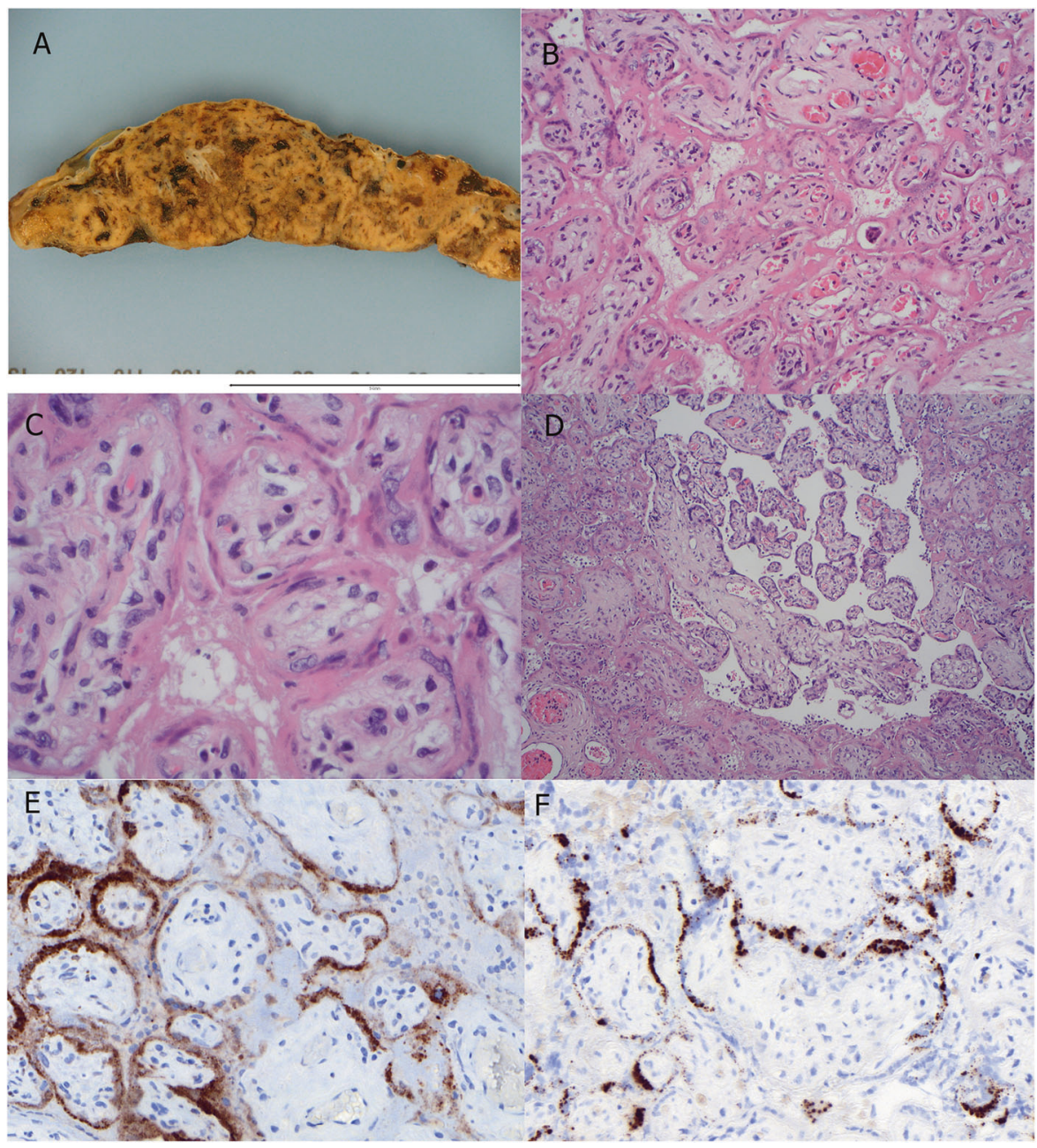

\begin{tabular}{llllllllll}
\hline Case & 1 & 2 & 3 & 4 & 5 & 6 & 7 & 8 & 9 \\
\hline Maternal age (yr) & 38 & 24 & 32 & 38 & 34 & 35 & 37 & 34 & 39 \\
Gestational age & 37.4 & 27.0 & 28.1 & 35.3 & 31.4 & 40.4 & 20.2 & 38.3 & 41.3 \\
Fetal death & Yes & Yes & Yes & No & Yes & No & Yes & No & No \\
Autopsy & Yes & Yes & No & No & Yes & No & Yes & No & No \\
Fetal weight (g) & 2935 & 781.5 & 950 & 2200 & 1580 & 3950 & 313 & 2580 & 3740 \\
Fetal gender & F & M & M & F & F & F & M & M & M \\
Placental disk weight (g) & 590 & 126 & 212 & 314 & 234 & 636 & 105 & 300 & 586 \\
SARS-CoV-2 RT-PCR & & & & & & & & & \\
Fetal tissues & Neg. & Neg. & & & Pos. & & & & \\
Fetal secretions & Pos. & & & & & Neg. & & & \\
Placenta & Pos. & Pos. & Pos. & Pos. & Pos. & Pos. & Pos. & Pos. & Pos. \\
SARS-CoV-2 IHC & & & & & & & & & \\
Fetal tissues & & Neg. & & & Neg. & & Neg. & & Pos. \\
$\begin{array}{l}\text { Placenta } \\
\text { Extent of placental lesions }\end{array}$ & Pos. & Pos. & Pos. & Pos. & Pos. & Pos. & Pos. & Pos. & Pos. \\
\hline
\end{tabular}

positive both for IHC and ISH (Fig. 2). Two of the focally affected placentas were the only that occurred in pregnancies negative for SARS-CoV-2 at admission for delivery.
Last positive test was obtained 19 days and 17 weeks before delivery, respectively. None of the 189 remaining placentas showed similar trophoblastic lesions. IHC for SARS-CoV-2 
Fig. 2 Focal trophoblastic damage. A In this grossly normal-looking placenta few small foci of intervillous space collapse were found as the two here depicted. Inset is shown at a higher magnification in (B) (H\&E, original magnification 10x). B Intervillous space collapse and trophoblastic necrosis looks quite similar to more extensive lesions depicted in Fig. 1 (original magnification, 20X). C Immunohistochemical staining for SARS-CoV-2 nucleoprotein shows staining in the injured villi but also in the surrounding normal-looking villi (original magnification 10X). D In situ hybridization with the RNAscope probe replicates the image of immunohistochemistry (original magnification 10x)

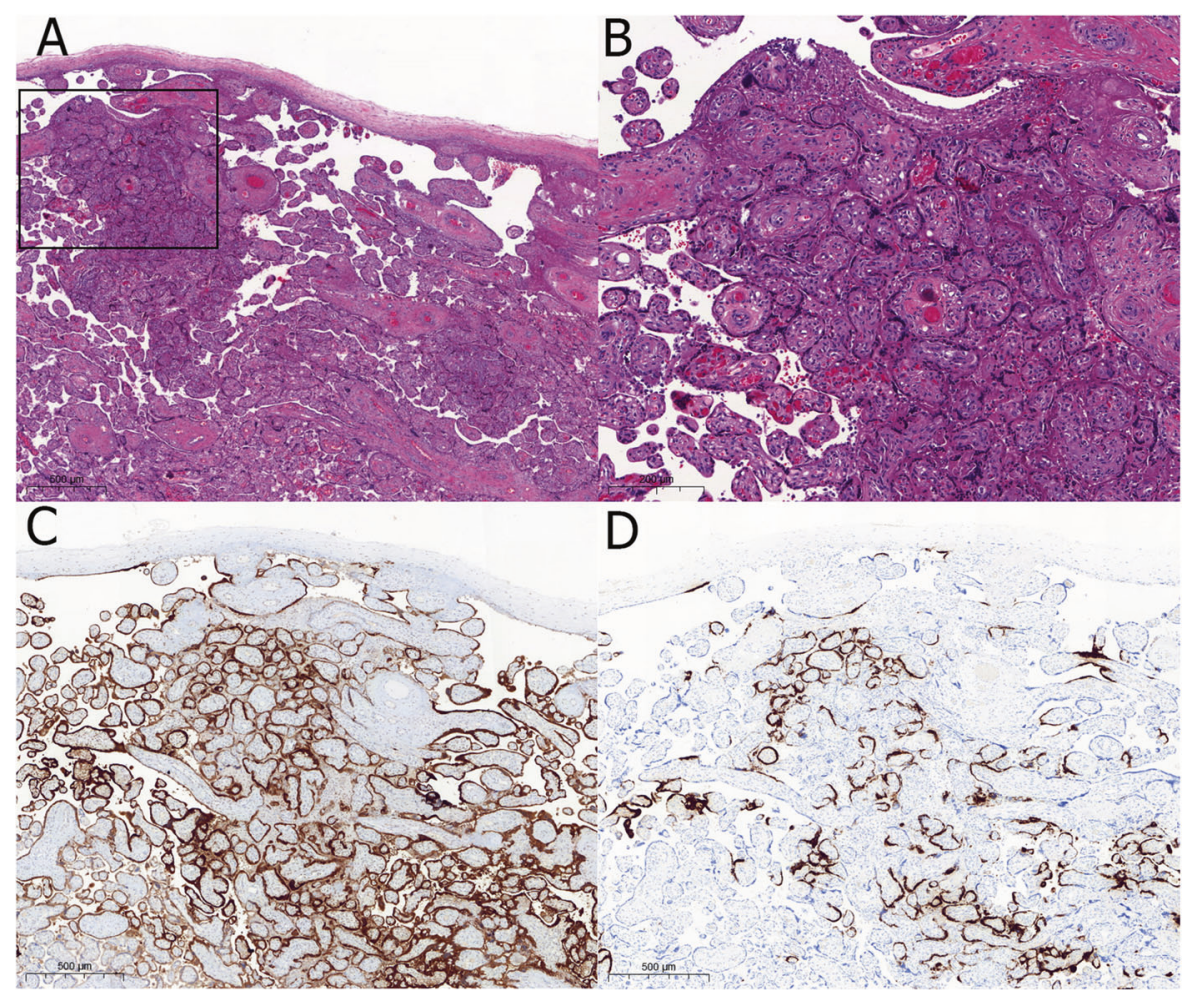

was negative in all of them. Twenty-four of these placentas were tested by RT-PCR giving negative results (Table 1).

Only an additional fetal loss was identified among the 189 negative placentas. It occurred in a second trimester SARS-CoV-2 actively infected pregnant woman. Placenta was small for gestational age and showed acute chorioamnionitis with severe stage 3 maternal response and nonsevere stage 1 fetal response. No signs of trophoblast necrosis were identified. RT-PCR and IHC of placenta were negative for SARS-CoV-2.

\section{Discussion}

Few diseases along human history generated an impact that could be compared to the COVID-19 pandemic. Serious concerns raised on COVID-19 consequences in pregnancy. Some early fetal losses were reported [2, 11, 12]. Series on placental pathology showed inconsistent results with little reproducibility among them. However, those series were mainly based in maternal SARS-Cov-2 positivity instead of placental infection $[3,4,6]$. Taken their results together it looked like placental lesions among COVID-19 affected pregnancies were not particularly different from nonaffected ones [6]. Besides, placental lesions had little, if any impact at all in newborn outcome in those series. Fetal losses in COVID-19 pregnancies were clinically attributed to SARS-CoV-2 infection, with two out of three tested cases having evidence of placental or amniotic fluid infection [2].
Vertical transmission or placental infection is supposed to occur at a low rate [1]. In fact, the existence of a "placental barrier" that prevents fetal infection was suggested [13].

SARS-CoV-2 infection in placenta was confidently proved in several cases with consistent results indicating that SARS-CoV-2 infects mainly trophoblastic cells outlining placental villi. Babies affected had an uneventful course and were discharged home in good health [7-9, 14]. Placental pathology was interpreted as inflammatory by most of the authors except for Debelenko et al., who focused their attention in the necrosis of the trophoblast and the acute and chronic component of the inflammatory infiltrate [7]. Our findings agree with this description. SARS-CoV-2-positive placentas show a particular lesion characterized by trophoblast necrosis and intervillous space collapse. It can be seen as tiny foci in the less affected cases or as massively extensive lesions in the more severe ones. When subtle, ancillary techniques for SARS-CoV-2 detection in placental tissues (IHC, ISH, RT-PCR) are required. Otherwise, they can be missed as ischemic lesions. Trophoblast necrosis can be found alone or in combination with intervillous inflammation and/or and intervillous fibrinoid deposition. The oppositeintervillous inflammation or intervillous fibrinoid deposition in the absence of trophoblast necrosis- was not found in any case, pointing to the trophoblast necrosis as the primary lesion in these placentas.

The combination of trophoblast necrosis and intervillous space collapse can be seen in ischemic lesions such as recent villous infarcts or villous agglutination (microinfarcts). Massive 
infarctions can be seen in abruptio placentae, but there were neither clinical nor pathological findings supporting this condition in the more severe cases, and the finding of spared small groups of villi surrounded by extensive necrotic areas and the relative sparing of the villous stroma in contrast with the extensive trophoblastic involvement go against an ischemic origin of necrosis.

The inflammatory infiltrate is reported as the main lesion in most of reports, but our findings do not support its leading role. First, although this infiltrate is present in all cases, it represents just a fraction of the injured tissue, being greatly surpassed by the extent of necrosis itself, even in the less severe cases. Moreover, the nature of the infiltrate, with and admixture of histiocytes and greater number of neutrophils than lymphocytes, resembles a reaction to necrosis, rather than its cause. Massive diffuse trophoblast necrosis is not a common finding in chronic intervillositis, whereas mixed inflammatory reaction is common in villous or trophoblastic necrosis. Although an increase in perivillous fibrin deposition was present in all cases, it was not a prominent feature in any of them. The macroscopic features resembling massive perivillous fibrinoid deposition could be explained by the intervillous space collapse that would produce both the tissue stiffness and the white stripes crossing the placental tissue, due to the absence of blood normally present in the intervillous space.

SARS-CoV-2 infection of trophoblastic cells could be the lesional mechanism in these cases. Sincytiotrophoblast express high levels (even higher than pneumocytes) of ACE2 , the receptor for SARS-CoV-2 cell entry in human cells $[13,15]$. Diffuse trophoblastic damage, with trophoblastic necrosis and intervillous space collapse with variable intervillous inflammatory infiltrates and perivillous fibrinoid deposition could be the placental counterpart of diffuse alveolar damage of the lung, which is the main histopathologic finding in COVID-19 pneumonia. A transcriptional pattern similar to that of SARS-CoV-2-infected lungs was found in samples of a SARS-CoV-2-infected placenta with a high viral load [16]. In lungs the virus is detected in the acute but not in the organizing phase of DAD, suggesting a major role for SARS-CoV-2 in initiating the acute lung injury [15]. The presence of the virus in trophoblast even in the earliest placental lesions would support this view.

This peculiar pattern of injury of diffuse trophoblastic damage with trophoblast necrosis plus intervillous space collapse with variable amounts of intervillous inflammation and perivillous fibrin deposition is either reported [79, 14, 16, 17] or identifiable in the pictures [2, 6, 18, 19] included in reports of SARS-CoV-2 infected placentas, but never in negative SARS-CoV-2 tested placentas. This strong association prompts us to consider it as a specific placental lesion in SARS-CoV-2 infection. Besides, it shows a high mortality that is mostly dependent on the extent of placental lesions, and it likely plays a role in vertical SARS-CoV-2 transmission, as two of the fetuses tested positive for SARS-CoV-2.

The identification of SARS-CoV-2 in placentas from pregnancies without evidence of current infection is an unexpected finding. It could be an example of persistent but localized infection. There is no way to rule out that the virus could remain hidden in small foci in patients after negativity of their nasopharyngeal swabs. Anyway, this finding seems of little significance for the pregnancy outcome.

In conclusion, our findings show a low rate of SARSCoV-2 placenta infection, confirm the pattern of lesion recently reported of placenta infection by SARS-CoV-2 and suggest that trophoblastic damage, characterized by either diffuse or focal trophoblast necrosis and intervillous space collapse with variable amounts of mixed intervillous inflammatory infiltrate and perivillous fibrinoid deposition, is the hallmark of SARS-CoV-2 infection of placenta. When diffuse, involving most of the chorionic tissue, it results in fetal demise. These findings should provide valuable tools for future investigations in the nature of maternal and fetal SARS-CoV-2 infection including pathogenetic mechanisms of injury and vertical infection transmission.

\section{Data availability}

Data available from the authors upon reasonable request.

Author contributions MG-P, AN, JC, JCF, and AN performed study concept and design, data acquisition, interpretation of data, writing, review, and revision of the paper. AN performed statistical analysis. FC, MA-G, AM-B, IG-R, BS, PG-A, AS participated in data acquisition. JH-L and MS provided technical support and S. RyC, provided material support. All authors read and approved the final paper.

Funding The authors received no specific funding for this work.

\section{Compliance with ethical standards}

Conflict of interest The authors declare no competing interests.

Ethics statement The article does not contain any studies with human participants or animals performed by any of the authors.

Publisher's note Springer Nature remains neutral with regard to jurisdictional claims in published maps and institutional affiliations.

\section{References}

1. Sharps MC, Hayes DJL, Lee S, Zou Z, Brady CA, Almoghrabi Y, et al. A structured review of placental morphology and histopathological lesions associated with SARS-CoV-2 infection. Placenta. 2020;101:13-29.

2. Richtmann R, Torloni MR, Otani ARO, Levi JE, Tobara MC. Silva $\mathrm{CdA}$, et al. Fetal deaths in pregnancies with SARS-CoV-2 infection in Brazil: a case series. Case Rep. Women's Heal. 2020;27:e00243. 
3. Baergen RN, Heller DS. Placental pathology in covid-19 positive mothers: preliminary findings. Pediatr Dev Pathol. 2020;23: 177-80.

4. Shanes ED, Mithal LB, Otero S, Azad HA, Miller ES, Goldstein JA. Placental pathology in COVID-19. Am J Clin Pathol. 2020; 154:23-32.

5. Smithgall MC, Liu-Jarin X, Hamele-Bena D, Cimic A, Mourad M, Debelenko L. et al. Third-trimester placentas of severe acute respiratory syndrome coronavirus 2 (SARS-CoV-2)-positive women: histomorphology, including viral immunohistochemistry and in-situ hybridization. Histopathology. 2020;77:994-9.

6. Hecht JL, Quade B, Deshpande V, Mino-Kenudson M, Ting DT, Desai N, et al. SARS-CoV-2 can infect the placenta and is not associated with specific placental histopathology: a series of 19 placentas from COVID-19-positive mothers. Mod Pathol. 2020;33:2092-103.

7. Debelenko L, Katsyv I, Chong AM, Peruyero L, Szabolcs M, Uhlemann A-C. Trophoblast damage with acute and chronic intervillositis: disruption of placental barrier by SARS-CoV-2. Hum Pathol. 2020;109:69-79.

8. Linehan L, O'Donoghue K, Dineen S, White J, Higgins JR, Fitzgerald B. SARS-CoV-2 placentitis: an uncommon complication of maternal COVID-19. Placenta. 2021;104:261-6.

9. Schoenmakers S, Snijder P, Verdijk RM, Kuiken T, Kamphuis SSM, Koopman LP, et al. Severe acute respiratory syndrome coronavirus 2 placental infection and inflammation leading to fetal distress and neonatal multi-organ failure in an asymptomatic woman. J Pediatric Infect Dis Soc. 2020. https://doi.org/10.1093/jpids/piaa153.

10. Khong TY, Mooney EE, Ariel I, Balmus NCM, Boyd TK, Brundler MA, et al. Sampling and definitions of placental lesions Amsterdam placental workshop group consensus statement. Arch Pathol Lab Med. 2016;140:698-713.
11. Baud D, Greub G, Favre G, Gengler C, Jaton K, Dubruc E, et al. Second-trimester miscarriage in a pregnant woman with SARSCoV-2 infection. JAMA. 2020;323:2198-200.

12. Hosier H, Farhadian SF, Morotti RA, Deshmukh U, Lu-Culligan A, Campbell KH, et al. SARS-CoV-2 infection of the placenta. J Clin Invest. 2020;130:4947-53.

13. Komine-Aizawa S, Takada K, Hayakawa S. Placental barrier against COVID-19. Placenta. 2020;99:45-9.

14. Vivanti AJ, Vauloup-Fellous C, Prevot S, Zupan V, Suffee C, Do Cao J, et al. Transplacental transmission of SARS-CoV-2 infection. Nat Commun. 2020;11:1-7.

15. Sauter JL, Baine MK, Butnor KJ, Buonocuore DJ, Chang JC, Jungbluth AA, et al. Insights into pathogenesis of fatal COVID-19 pneumonia from histopathology with immunohistochemical and viral RNA studies. Histopathology. 2020;77:915-25.

16. Cribiù FM, Erra R, Pugni L, Rubio-Perez C, Alonso L, Simonetti S, et al. Severe SARS-CoV-2 placenta infection can impact neonatal outcome in the absence of vertical transmission. J Clin Invest. 2021;131:e145427.

17. Sisman J, Jaleel MA, Moreno W, Rajaram V, Collins RRJ, Savani $\mathrm{RC}$, et al. Intrauterine transmission of SARS-COV-2 infection in a preterm infant. Pediatr Infect Dis J. 2020;39:265-7.

18. Mongula JE, Frenken MWE, van Lijnschoten G, Arents NLA, De Wit-Zuurendonk LD, Schimmel-De, et al. COVID-19 during pregnancy: non-reassuring fetal heart rate, placental pathology and coagulopathy. Ultrasound Obstet Gynecol. 2020;56:773-6.

19. Facchetti F, Bugatti M, Drera E, Tripodo C, Sartori E, Cancila V, et al. SARS-CoV2 vertical transmission with adverse effects on the newborn revealed through integrated immunohistochemical, electron microscopy and molecular analyses of Placenta. EBioMedicine. 2020;59:102951. 